\title{
Magnetic Films on Nanoparticle Arrays
}

\author{
Manfred Albrecht ${ }^{*}$ and Denys Makarov ${ }^{\#}$
}

Institute of Physics, Chemnitz University of Technology, 09107 Chemnitz, Germany

\begin{abstract}
Novel properties originating from the combination of magnetism with the reduced dimensionality of nanoobjects are of considerable interest for fundamental science but are also readily used in a wide range of technological applications. To fulfill the demand for larger capacities of storage systems, complex magnetic heterostructures of a nanometer size are envisioned as future magnetic storage media. Here we review recent advances on a novel gradient nanomaterial fabricated by deposition of magnetic thin films onto self-assembled arrays of nonmagnetic spherical particles, resulting in arrays of magnetic caps. Due to the curvature of the particle's surface, magnetic and structural properties of the magnetic thin films differ substantially from their planar counterparts with respect to the equilibrium magnetic domain pattern as well as magnetization reversal behavior. It will be shown that varying the size of the particles as well as the thickness of the deposited layers, the magnetostatic and exchange coupling between the neighboring caps can be systematically modified, providing direct access to the change of fundamental magnetic interactions at the nanoscale. Furthermore, these magnetic cap structures were employed to realize so called bit patterned magnetic media, which is one of the most promising concepts in magnetic data storage to provide areal densities beyond $1 \mathrm{Tbit}_{/ \mathrm{inch}}{ }^{2}$. In this respect, important aspects regarding the potential application of the magnetic cap structures in magnetic data storage will be addressed.
\end{abstract}

Keywords: Nanomagnets, self-assembly, perpendicular magnetic anisotropy, magnetization reversal, magnetic coupling, magnetic data storage.

\section{INTRODUCTION}

The physical as well as chemical properties of nanoscale objects differ substantially from their bulk counterparts, thus resulting in a variety of applications in the field of catalyst, sensorics, magnetic recording, biology, and medicine [1-7]. The most prominent examples are the tremendous increase in areal density of magnetic recording $[8,9]$, creation of high efficiency hybrid solar cells [2, 10], and sensoric applications for pathogen detection $[4,11]$, to name a few. This remarkable success is mainly due to specific novel optical and electromagnetic properties that nanostructures exhibit as well as their adoptability to the needs of applications. In this respect, novel properties of magnetic nanostructures can arise for a number of reasons, such as confinement of the magnetic material into two- or one-dimensional structures and close proximity with other materials in layered or nanocomposite architectures.

One of the driving forces for the development of innovative magnetic materials is the magnetic recording industry. In conventional hard disk drives (HDDs) an individual bit consists of weakly magnetically coupled $\mathrm{CoCrPt}$ grains [12]. To further increase the areal storage density (number of bits per unit area) a reduction of the dimensions of the grains is required. However, this reduction has a lower boundary, as with reduction of the grain size, the

*Address correspondence to this author at the Institute of Physics, Chemnitz University of Technology, 09107 Chemnitz, Germany; Tel: +49 (0) 371531 36831; Fax: +49 (0) 371531 21619;

E-mail:manfred.albrecht@physik.tu-chemnitz.de

${ }^{\#}$ Current Address: Institute for Integrative Nanosciences, IFW Dresden Helmholtzstr. 20, D-01069 Dresden, Germany. magnetic anisotropy energy $K_{\mathrm{U}} \cdot V\left(K_{\mathrm{U}}\right.$ being the magnetic anisotropy constant and $V$ being the volume of a grain) will come closer to the thermal activation energy. Thus, the recording media reaches the superparamagnetic limit and becomes thermally instable [13]. Estimates show that the present perpendicular recording concept based on granular CoCrPt-oxide media will face the fundamental superparamagnetic limitation at areal densities of about $1 \mathrm{Tbit} / \mathrm{inch}^{2}$ (current products have areal density of about $650 \mathrm{Gbit} / \mathrm{inch}^{2}$ ). Therefore, in order to keep the same growth rate, novel recording concepts are required. In this respect, the most promising is to use lithographically defined media, namely bit patterned media (BPM), which is expected to extend the storage density beyond $1 \mathrm{Tbit}^{\mathrm{inch}}{ }^{2}[14,15]$. In the BPM concept, an individual bit will be stored in a single magnetic nanostructure. Candidates for a recording layer in BPM are films with strong out-of-plane magnetic anisotropy, i.e. $[\mathrm{Co} / \mathrm{Pt}(\mathrm{Pd})]_{\mathrm{N}}$ multilayers [15] as well as hard magnetic $\mathrm{FePt}$ alloys $[9,16,17]$. For the latter, uniaxial magnetic anisotropy of chemically $L 1_{0}$ ordered FePt alloys with equiatomic composition is found to be about $70 \times 10^{6} \mathrm{erg} / \mathrm{cm}^{3}$ [18]. This allows satisfying the criteria of thermal stability for magnetic nanostructures with sizes down to about $3 \mathrm{~nm}$, resulting in an enormous storage density of about $50 \mathrm{Tbit}^{- \text {inch }^{2}}$ [19]. In this respect, considerable efforts have been dedicated to fabricate ordered arrays of magnetic nanostructures using various lithography techniques (Fig. 1a) [20-23] including nanoimprint approaches [24, 25]. However, most of these methods may not be suitable for large-scale device fabrication because they are multistep, expensive, and involve time-consuming procedures. Other ways to produce patterned templates are self-assembly techniques such as anodisation [26], electro-assisted deposition [27], 
(a) Co/Pd multilayers on e-beam fabricated pattern (b) Co/Pd multilayers on Si pillars fabricated via directed assembly of block copolymer
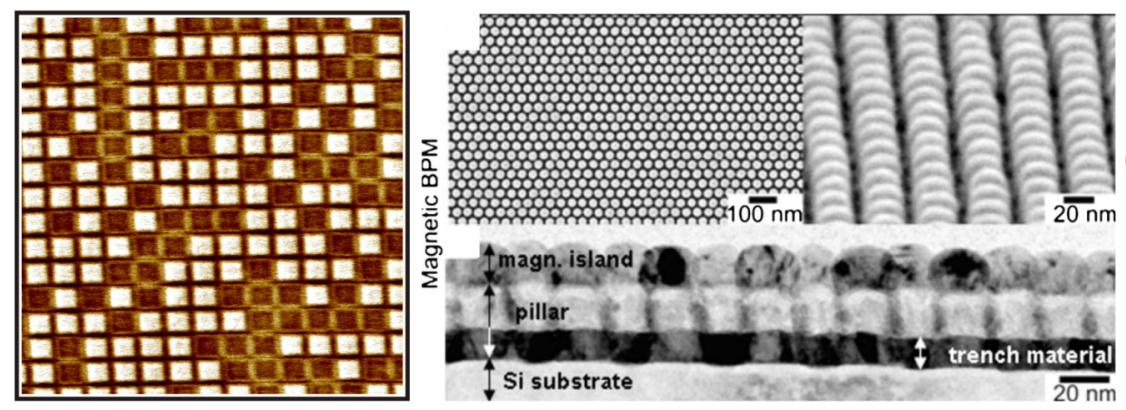

(c) Self-assembly

of FePt nanoparticles

Fig. (1). (a) Magnetic force microscopy (MFM) image revealing the magnetic state in the array of $[\mathrm{Co} / \mathrm{Pd}]$ islands with a size of $50 \mathrm{~nm}$ prepared by e-beam patterning [20]. Reprinted with permission from Hu et al. [20]. Copyright 2004, American Institute of Physics. (b) BPM array consisting of $[\mathrm{Co} / \mathrm{Pd}]$ multilayers deposited onto Si pillar substrates fabricated via e-beam directed assembly of block copolymer films [34]. Left panel: top view scanning electron microscopy (SEM) micrograph. Right panel: section view at $85^{\circ}$ angle. Bottom: Bright field transmission electron microscopy (TEM) cross-sectional image. Reprinted with permission from Hellwig et al. [34]. Copyright 2010, American Institute of Physics. (c) Self-assembly of fcc disordered FePt nanoparticles with a size of about $5 \mathrm{~nm}$ [7].

evaporation-induced self-assembly associated to liquid deposition [28-32], phase separation in block copolymers (Fig. 1b) [33, 34], surface instability induced by ion bombardment [35], or self-assembled magnetic particles (Fig. 1c) $[7,36]$.

An alternative method, which combines self-assembly of nonmagnetic spherical nanoparticles with defined magnetic properties provided by a magnetic film deposited onto the particles, was recently introduced [37]. This route provides an elegant way to create magnetic nanostructure arrays (cap arrays) with strong PMA as required for high thermal stability [38-44]. In these systems, the fundamental magnetic interactions (exchange and magnetostatic) can be easily tuned by varying the particle diameter as well as the material properties of the deposited magnetic films. For instance, changing the size of the particles allows studies of the scaling dependence of the integral magnetic properties like coercive field and switching field distribution.

In the following we summarize recent results on the magnetic behavior of magnetic thin films grown onto assemblies of nonmagnetic spherical particles of various sizes. Due to the curvature of the particle's surface, the structural and magnetic properties of the individual magnetic caps as well as their arrays are modified with respect to their planar counterparts and thus magnetic cap structures on particles reveal a peculiar magnetization reversal behavior. Furthermore, important aspects regarding the potential application of the considered system in magnetic data storage will be addressed.

\section{FABRICATION OF SELF-ASSEMBLED ARRAYS OF NONMAGNETIC SPHERICAL PARTICLES}

Preparation of nonmagnetic particle monolayers of various diameters ranging from 10 up to $900 \mathrm{~nm}$ was carried out following a routine initially proposed by Micheletto et al. [45]. In this case, a droplet of a particle/water solution is deposited onto a cleaned thermally oxidized $\mathrm{Si}(100)$ wafer. The cleaning involves ultrasonication in acetone, ethanol and purified water followed by treatment in oxygen plasma for 4 min. The evaporation process of the droplet takes place in a small and tilted box (Fig. 2a), leading to the growth of the particle monolayers. By varying particle concentration in the colloid solution, the tilting angle and the size of the droplet, a sufficient coverage of the substrate with particle monolayers is possible (Fig. $\mathbf{2 b}, \mathbf{c}$ ).

To increase the degree of order in the self-assembly process, Kappenberger et al. [42] employed templatedirected self-assembly to arrange spherical particles into a polymer resist template with regular hole arrays, produced using extreme ultraviolet interference lithography (EUV-IL) at the XIL beamline of the Swiss Light Source. The fabricated patterns consist of arrays of holes formed in a thin polymethyl methacrylate (PMMA) resist layer on a $\mathrm{Si}$ substrate. The substrate is then tilted by $15^{\circ}$ and a colloidal

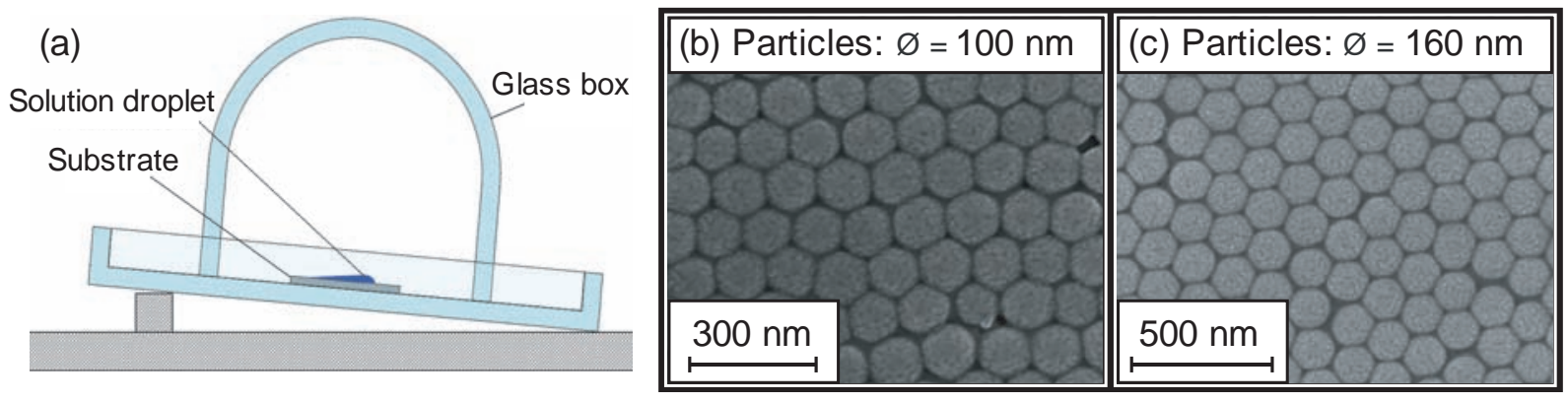

Fig. (2). (a) Schematics of the set-up used for preparation of self-assemblies of nonmagnetic spherical particles. Self-assembly of $\mathrm{SiO}_{2}$ particles with nominal size of (b) $100 \mathrm{~nm}$ and (c) $160 \mathrm{~nm}$. 
solution consisting of sulphatized polystyrene nanoparticles diluted with Milli-Q water is dropped onto the substrate so that the nanoparticles are trapped in the template holes as the solution flows slowly downwards as illustrated in Fig. (3). As the colloidal solution dries, the nanoparticles are fixed inside the holes, and a dense long-range order is formed. A typical patterned hole array in a PMMA resist with a period of $100 \mathrm{~nm}$ on a Si substrate is shown in Fig. (4a). The hole size is $60 \mathrm{~nm}$ with a resist thickness of $25 \mathrm{~nm}$. Nanoparticles with an average diameter of $60 \mathrm{~nm}$ which have been captured in the holes to form a regular square lattice are shown in Fig. (4b). This template-directed ordering method proved to be very tolerant to dispersion in nanoparticles size, and we were able to achieve long-range ordering of the colloidal nanoparticles over the full template area $\left(5 \times 5 \mu \mathrm{m}^{2}\right)$.

(a)

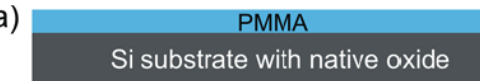

(b)

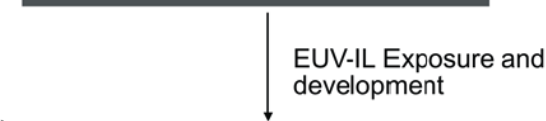

(c)
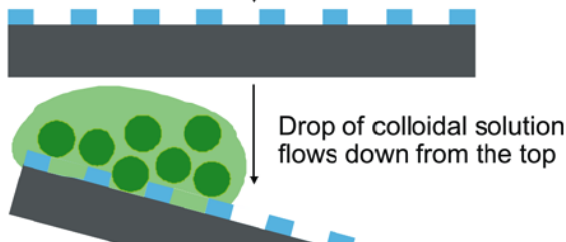

(d)

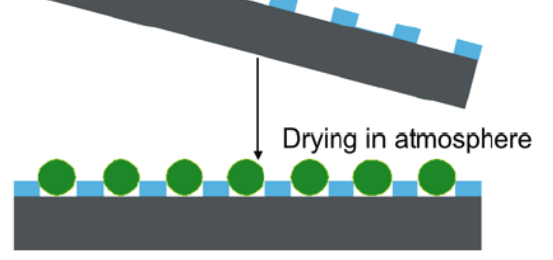

Fig. (3). (a)-(d) Schematics revealing the template-directed assembly process.
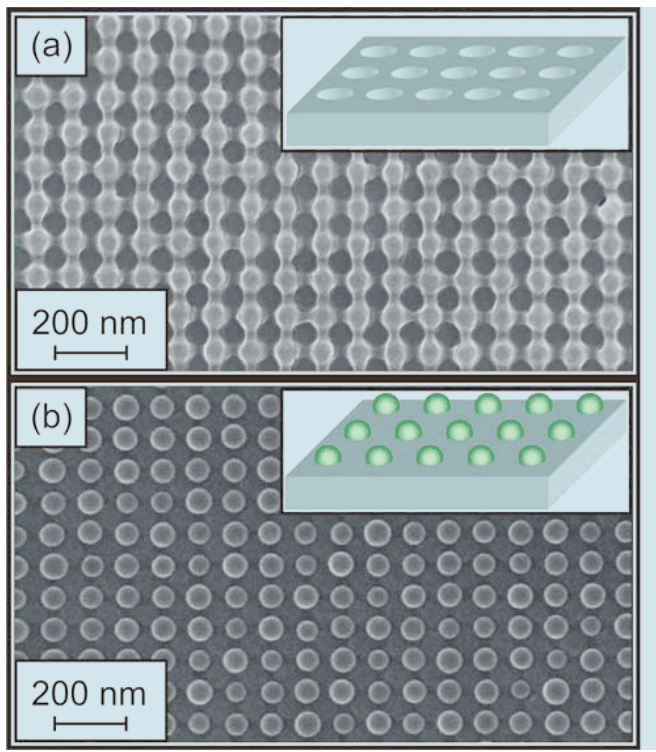

Further attempts to apply this template-directed assembly process to order even smaller nanoparticles with a diameter of $20 \mathrm{~nm}$ in regular hole arrays with periods down to $42 \mathrm{~nm}$ and with hole size as small as $22 \mathrm{~nm}$ were carried out. Also for this case the template assisted self-assembly process works well although not perfectly (Fig. 4c, d).

\section{3. $[\mathrm{Co} / \operatorname{Pt}(\mathrm{PD})]_{\mathrm{N}}$ MULTILAYER STACKS ON ARRAYS OF SPHERICAL PARTICLES}

$[\mathrm{Co} / \mathrm{Pt}(\mathrm{Pd})]_{\mathrm{N}}$ multilayers are a convenient model system to investigate the specific magnetic and structural properties of magnetic thin films grown on nanoparticle arrays. In particular, the magnetic anisotropy can be easily varied by adjusting the $\mathrm{Co}$ individual layer thickness. $[\mathrm{Co} / \mathrm{Pt}(\mathrm{Pd})]_{\mathrm{N}}$ multilayers exhibit perpendicular magnetic anisotropy (PMA) in a certain range of Co thicknesses where the interface anisotropy is large enough to overcome the shape anisotropy [46]. With increasing Co layer thickness, a socalled thickness-driven spin reorientation transition (SRT) occurs, where the direction of the magnetic easy axis changes into the film plane. For the crossover Co layer thickness, $t_{\mathrm{SRT}}$, values of $(0.3-1.7) \mathrm{nm}$ have been reported [47-50], depending on the choice of the substrate [48], the Pt layer thickness, and the interfacial roughness [49].

Growth of $[\mathrm{Co} / \mathrm{Pt}(\mathrm{Pd})]_{\mathrm{N}}$ multilayers on curved substrates results in a tilt of the easy axis of magnetization following the curvature of the particle surface. The presence of the tilt of easy axis is expected to substantially influence the equilibrium domain pattern and to modify the magnetization reversal behavior as was investigated in $[\mathrm{Co} / \mathrm{Pt}]_{\mathrm{N}}$ multilayer systems with varying individual Co layer thickness grown on assemblies of polystyrene particles with various particle sizes. First hint regarding such a modification comes from the detailed analysis of the angular dependence of the switching field of magnetic cap structures [38]. Fig. (5) shows the angular dependence of the normalized switching

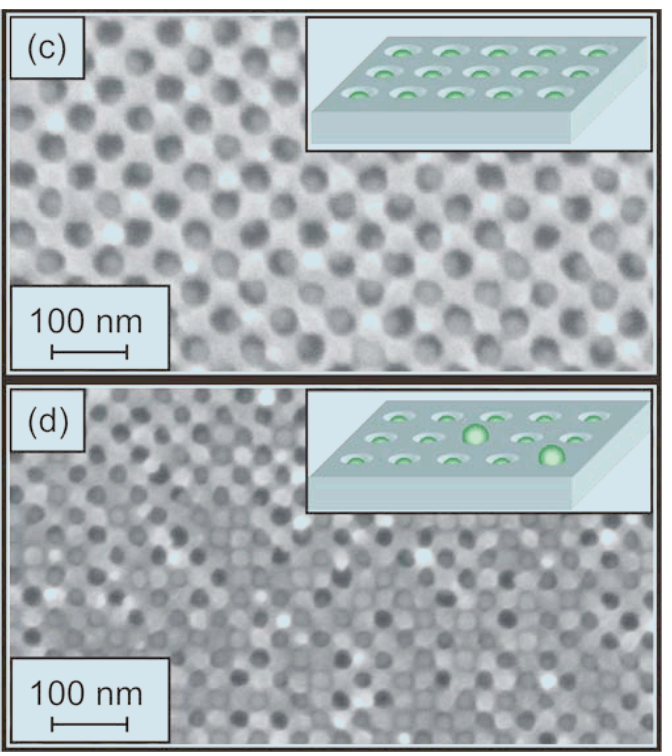

Fig. (4). SEM images of (a) a patterned hole array in a PMMA resist created with EUV-IL with a hole size of $60 \mathrm{~nm}$ and a period of $100 \mathrm{~nm}$. (b) Nanospheres with a diameter of $60 \mathrm{~nm}$ captured in the regular square hole array shown in (a). Template-directed self-assembly of $20 \pm 5$ $\mathrm{nm}$ nanospheres in (c) template with a hole diameter of $30 \mathrm{~nm}$ and a period of $50 \mathrm{~nm}$ and (d) template with a hole diameter of $22 \mathrm{~nm}$ and a period of $42 \mathrm{~nm}$. The ordering is schematically shown in the insets. Reprinted with permission from Kappenberger et al. [42]. Copyright 2009, American Institute of Physics. 
field, $H_{\mathrm{S}}$, measured by polar magneto-optical Kerr effect (MOKE) magnetometry. After a rapid decrease for small angles $\theta$, the magnitude of the switching field for the caps on the $50 \mathrm{~nm}$ particles has a broad minimum from $45^{\circ}$ to $80^{\circ}$ [37]. Reversal studies on larger particles reveal a different behavior, showing a little drop in $\mathrm{H}_{\mathrm{S}}$ for the $110 \mathrm{~nm}$ particles or a small increase for the $310 \mathrm{~nm}$ particles towards higher angles, indicating a domain-wall-motion controlled reversal mechanism. This behavior differs substantially from the Stoner-Wohlfarth (S-W) model, plotted as a solid line in Fig. (5b), which is applicable to single-domain particles with uniaxial anisotropy reversing via coherent rotation [51].

To clarify this point, a series of micromagnetic simulations was performed. Details on the simulation procedure can be found in $[38,43,44]$. In order to implement the properties of the magnetic caps, the volume was divided into a mesh of finite elements with a maximum side length of $5 \mathrm{~nm}$. For $0^{\circ}<\theta<50^{\circ}$ the magnitude of magnetic anisotropy, saturation magnetization and exchange constant of the individual elements is set to constant values, $K_{\mathrm{U} 0}, M_{\mathrm{S} 0}$, and $A_{0}$, respectively, since the thickness of the single Co layers changes from about 1.5 to 1 monolayer. To account for the change of the anisotropy with Co thickness, $K_{\mathrm{U} 0}$ has to be considered as an effective value $K_{\mathrm{U}}$, which will be reduced compared to the value measured on a plane reference film sample. For higher angles both anisotropy and saturation magnetization are approximated by a Gaussian function $f(\theta)=\exp \left[-\left(\left(\theta-\theta_{0}\right) / \sigma_{0}\right)^{2}\right]$ with $\theta_{0}=50^{\circ}$ and $\sigma_{0}=0.1$, leading to a steep drop in $K_{\mathrm{U}}(\theta)$ and $M_{\mathrm{S}}(\theta)$ to zero. Note that the anisotropy distribution is pointing perpendicular to the particle surface and its absolute value is assumed to be independent of particle size as long as the particle diameter is larger than the grain size of the deposited film. A schematic cross section of the model is depicted in Fig. (5a) sketching the distribution of the anisotropy orientation and also its local magnitude variation.

Single particle hysteresis loops were simulated for different particle sizes and various directions of the external field with $A_{0}=1 \times 10^{-11} \mathrm{~J} / \mathrm{m}$ and $M_{\mathrm{S} 0}=0.5 \mathrm{MA} / \mathrm{m} . K_{\mathrm{U} 0}$ is the fitting parameter for the simulations. The normalized best fit results using one single $K_{\mathrm{U} 0}$ value are compared to the experimental data and presented together in Fig. (5a). Best fits were obtained with $K_{\mathrm{U} 0}=0.3 \mathrm{MJ} / \mathrm{m}^{3}$. For all particle sizes there is good agreement between simulations and experiment, clearly verifying the assumptions. To prove the importance of the anisotropy distribution, additional simulations were performed by applying a uniaxial anisotropy perpendicular to the substrate surface for all finite elements. All other parameters were unaltered. This model and the results of the simulations are given in Fig. (5b), which does not fit the experimentally observed behavior.

To investigate the influence of the curvature of the surface on the spin-reorientation transition in this novel gradient nanomaterial, a series of $\left[\mathrm{Co}\left(t_{\mathrm{Co}}\right) / \operatorname{Pt}(0.8 \mathrm{~nm})\right]_{8}$ multilayers with various individual Co layer thicknesses $t_{\mathrm{Co}}$ between 0.2 and $0.8 \mathrm{~nm}$ was evaporated at room temperature onto the particle arrays with different particle sizes [52]. The growth of the multilayer was assisted by a $2.9 \mathrm{~nm}$ thick $\mathrm{Pt}$ buffer layer. To prevent oxidation, the stack was covered by an additional $0.8 \mathrm{~nm}$ thick $\mathrm{Pt}$ layer. In addition, continuous $[\mathrm{Co} / \mathrm{Pt}]_{8}$ multilayer films were grown under identical conditions on flat $\mathrm{Al}_{2} \mathrm{O}_{3}(0001)$ substrates for comparison.
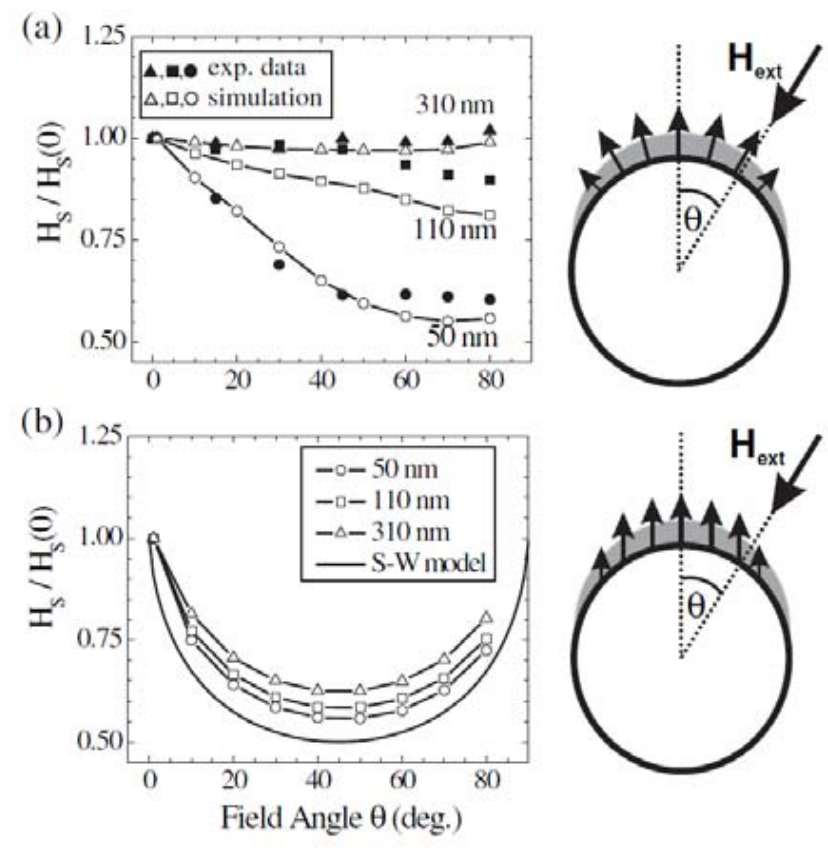

Fig. (5). Normalized switching field as a function of applied external field angle $\theta$ (a) from experimental data and simulations with spatial anisotropy distribution for all particle sizes and (b) from simulations for caps assuming uniaxial anisotropy, including a representation of the S-W model [51]. The solid lines serve as a guide to the eye for the simulated data points. Included are schematics of the magnetic caps showing the local anisotropy distribution and the external field direction. Reprinted with permission from Ulbrich et al. [38]. Copyright 2006, American Physical Society.

The magnetization reversal process was analyzed by polar MOKE for different particle sizes and for various Co layer thicknesses (Fig. 6). It is apparent that $[\mathrm{Co} / \mathrm{Pt}]$ multilayers of up to an individual Co layer thickness of about $0.6 \mathrm{~nm}$ have their easy axis of magnetization pointing perpendicular to the substrate plane and reveal a ratio between the remanence and saturation magnetization of almost one, independent of the particle size. With decreasing particle size the coercivity increases for samples with the same $[\mathrm{Co} / \mathrm{Pt}]$ stack as summarized in Fig. (6c). The largest coercivity is observed for a Co layer thickness of $0.3 \mathrm{~nm}$ and decreases almost linearly with increasing Co layer thickness for all particle sizes resembling the increase of the shape anisotropy of the multilayer stack favoring the in-plane orientation of the easy axis of magnetization. For the smallest Co layer thickness of $0.2 \mathrm{~nm}$ a reduced coercivity is observed most likely due to incompletion of a continuous Co layer, which will reduce the anisotropy and thus is more viable to thermal activation processes. A similar behavior has also been observed for continuous films (Fig. 6b, c). In addition, superconductive quantum interference device hysteresis measurements have been performed on these film samples to extract the effective magnetic anisotropy, revealing a transition of easy axis orientation from perpendicular to the film plane to an in-plane direction at a Co layer thickness of about $0.8 \mathrm{~nm}$. These results are consistent with experimental data reported in the literature $[46,53]$. After $\mathrm{SRT}$ at $t_{\mathrm{Co}}=0.8 \mathrm{~nm}$, remanence magnetization measured in 
the out-of-plane direction decreases (Fig. 6) as the easy axis of magnetization turns into the film plane.

Investigation of the local magnetic domain patters using magnetic force microscopy (MFM) at remanence reveals that a single-domain magnetic state is the most favorable state for a Co layer thickness of up to $0.4 \mathrm{~nm}$ for all particle sizes; larger Co layer thicknesses results in a multi-domain state in magnetic caps [52].

One of the key issues, limiting the applicability of the BPM concept, is the substantial broadening of the switching field distribution (SFD) for arrays of magnetic nanoparticles. The main contribution to the SFD is given in our case by the particle size distribution. However, at small separation distances between nanostructures, the dipole-dipole interaction becomes rather important strongly influencing the magnetization reversal in nanopatterned media [54]. Therefore, a detailed study of the influence of the magnetostatic coupling on the magnetization reversal of $[\mathrm{Co} / \mathrm{Pd}]_{\mathrm{N}}$ multilayer stacks grown on nanoparticle arrays will be discussed in the following [43].

For this study we covered assemblies of polystyrene particles (sizes: 58 and $110 \mathrm{~nm}$ ) with $[\mathrm{Co} / \mathrm{Pd}]_{\mathrm{N}}$ multilayer stacks with different number of bilayers, $N$, ranging between 8 and 80 . The deposition of $[\operatorname{Co}(0.27 \mathrm{~nm}) / \operatorname{Pd}(0.8 \mathrm{~nm})]_{\mathrm{N}}$ multilayer stacks was performed by magnetron sputtering. In order to improve the growth conditions of the multilayers, a 5-nm-thick Pd buffer layer was sputtered onto a 1-nm-thick $\mathrm{Cr}$ seed layer grown directly on the particles. A 1-nm-thick Pd capping layer was additionally deposited to protect the sample from oxidation. In addition, a series of reference samples was prepared on planar glass substrates for comparison.

An additional sample, $[\mathrm{Co}(1.8 \mathrm{~nm}) / \operatorname{Pd}(1.8 \mathrm{~nm})]_{8}$, was prepared on nominally $50 \mathrm{~nm} \mathrm{SiO}_{2}$ particles for Transmission Electron Microscopy (TEM) investigation. A crosssection bright field TEM image taken on this sample is shown in Fig. (7). Although the curved surface of a spherical particle results in a tilt of the growth direction of the metallic film, a well-defined multilayer stack is observed up to the angle of $40^{\circ}$ with respect to the symmetry axis of the caps. A similar study was also reported by Soares et al. [55].

In addition, the deposition of a metallic film on a curved substrate leads to the formation of grains with tilted growth direction and with lateral grain sizes which become smaller with tilt angle. The presence of the tilt in the growth direction of the magnetic multilayer has important consequences on the magnetic properties of the $[\mathrm{Co} / \mathrm{Pd}]_{\mathrm{N}}$ multilayer stack: As the out-of-plane easy axis of magnetization in $[\mathrm{Co} / \mathrm{Pd}]$ multilayers is driven by the interface anisotropy $[56$, 57], a tilt in the growth direction of the stack should result in a deviation of the easy axis following the curvature of the particle's surface. This idea, as was already mentioned, was utilized to explain the modification of the magnetization reversal process in $[\mathrm{Co} / \mathrm{Pt}(\mathrm{Pd})]_{\mathrm{N}}$ multilayers grown on nanoparticles [37, 38].

Another important aspect is that with increasing angle between the deposition direction and the normal to the particle surface the growth of a multilayer stack results in a

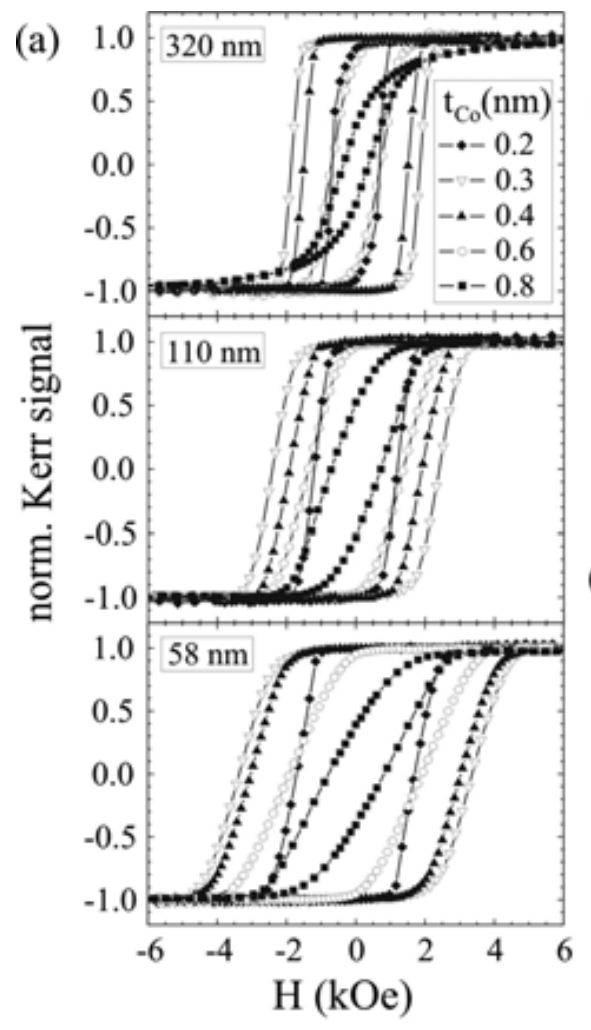

(b)

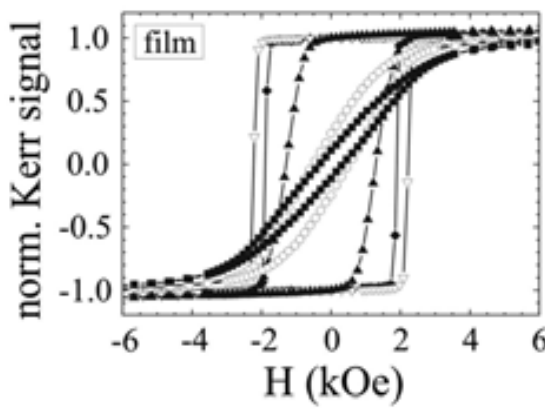

(c)

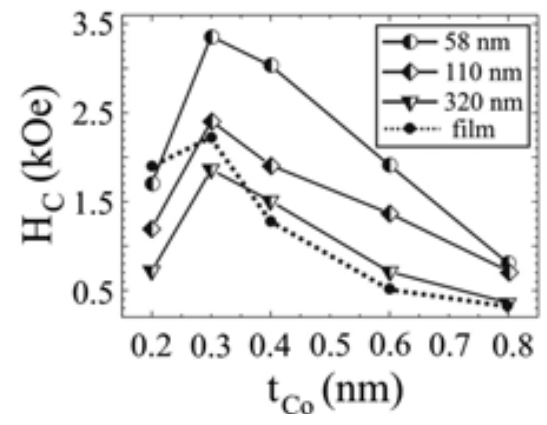

Fig. (6). (a) MOKE hysteresis loops of $\left[\mathrm{Co}\left(t_{\mathrm{Co}}\right) / \mathrm{Pt}(0.8 \mathrm{~nm})\right]_{8}$ film stacks with various Co layer thicknesses $t_{\mathrm{Co}}$ deposited onto particle arrays with sizes between 58 and $320 \mathrm{~nm}$. (b) For comparison the same film stack was also deposited on a flat $\mathrm{Al}_{2} \mathrm{O}_{3}(0001)$ substrate. (c) Coercive field $H_{\mathrm{C}}$ as a function of individual Co layer thickness for various particle sizes and for the continuous films. Reprinted with permission from Ulbrich et al. [52]. Copyright 2008, American Institute of Physics. 

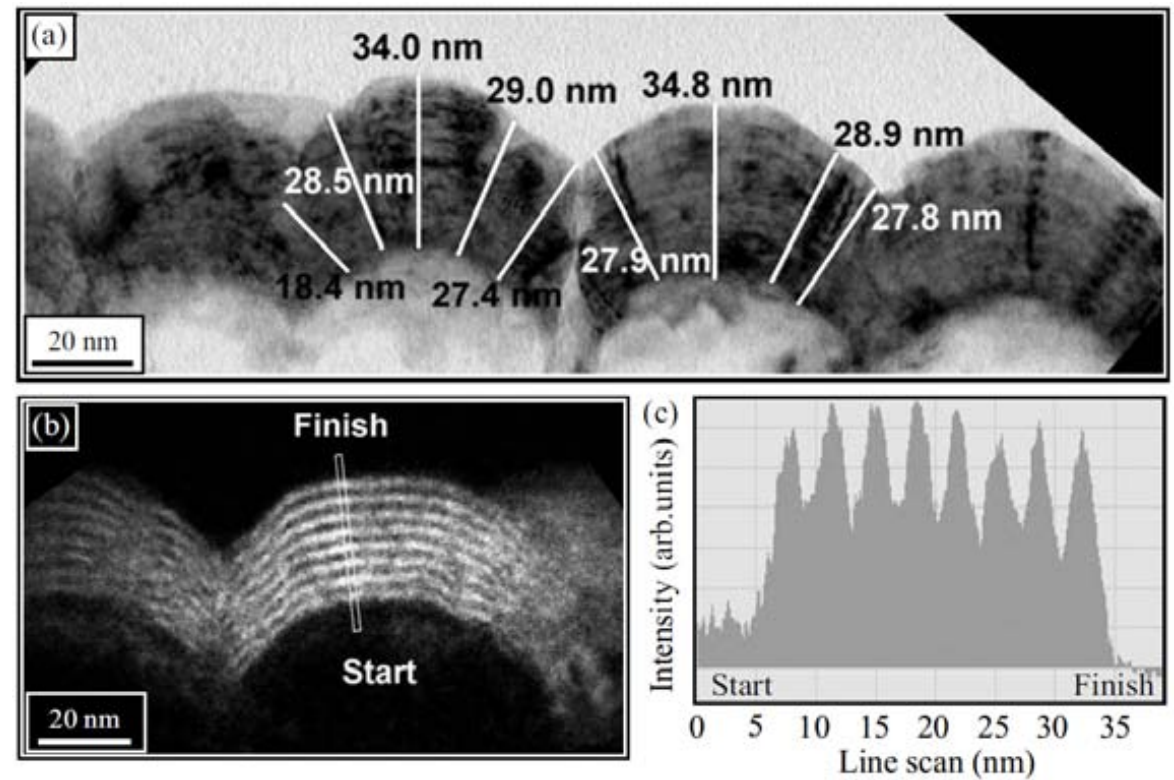

Fig. (7). TEM images of an assembly of $\mathrm{SiO}_{2}$ particles with a size of nominally $50 \mathrm{~nm}$ covered with a $[\mathrm{Co}(1.8 \mathrm{~nm}) / \mathrm{Pd}(1.8 \mathrm{~nm})]_{8} \mathrm{multilayer}$ stack: (a) bright-field cross-sectional TEM image. The thickness variation in the film with varying angle is indicated. (b) Energy filtered image (only Co layers are visible as the bright layers). (c) Intensity profile taken along the line marked in (b). Reprinted with permission from Ulbrich et al. [43]. Copyright 2010, American Physical Society.

mixture of $\mathrm{Co}$ and $\mathrm{Pd}$ species. This degradation of the multilayer structure can be seen in the TEM image of Fig. (7b) in particular at an angle of above $40^{\circ}$. For the case of $[\mathrm{Co}(0.27 \mathrm{~nm}) / \mathrm{Pd}(0.8 \mathrm{~nm})]_{\mathrm{N}}$ multilayers with a rather thin $\mathrm{Co}$ layer, the formation of a Pd-rich Co-Pd alloy with a Curie temperature below $300 \mathrm{~K}$ is expected $[58,59]$. In this case, although the neighboring nanocaps are interconnected, the intersection region is expected to be paramagnetic leading to exchange isolation of the neighboring caps in the array [37, 60] as illustrated in Fig. (8).

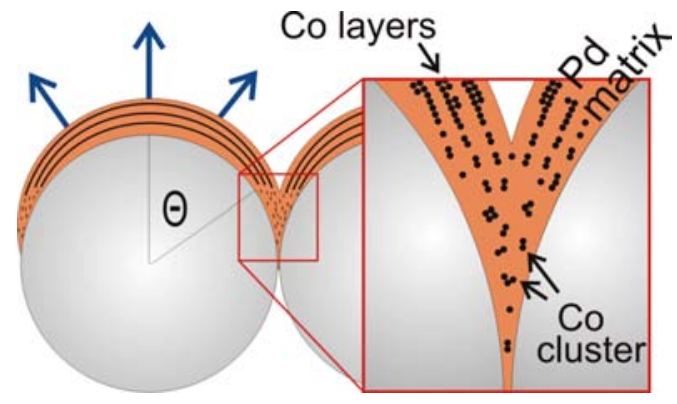

Fig. (8). Schematics revealing the transition from the well-defined $[\mathrm{Co} / \mathrm{Pd}]_{\mathrm{N}}$ multilayers to the Pd-rich Co-Pd alloy resulting in strong exchange decoupling of the neighboring cap structures in the array. Reprinted with permission from Moser et al. [60]. Copyright 2010, American Institute of Physics.

Hysteresis loops measured on $[\mathrm{Co} / \mathrm{Pd}]$ multilayers grown on assemblies of particles are presented in Fig. $(\mathbf{9 a}, \mathbf{b})$. A systematic shearing of the hysteresis loops with increasing number of bilayers is observed indicating the influence of the dipolar fields originating from neighboring magnetic caps. Furthermore, the variation of $H_{\mathrm{C}}$ in the films grown on the particle assemblies with the number of bilayers is different compared to the observation on the planar substrates. Thus, $[\mathrm{Co} / \mathrm{Pd}]$ multilayers on assemblies of particles with a diameter of $110 \mathrm{~nm}$ possess a rather constant coercivity independent of the number of bilayer $N$ (Fig. 9a). This might be related to the different microstructure of a multilayer stack grown on particles compared to planar substrates. The absolute value of coercive field is however close to the one observed on the planar substrates. In the case of the films grown on $58 \mathrm{~nm}$ particles (Fig. 9b) a remarkable increase of the out-of-plane coercivity compared to the deposits on $110 \mathrm{~nm}$ particles (Fig. 9a) as well as on the planar substrates is observed for the smallest bilayer number. However, with the increase of the bilayer number, the coercivity gradually decreases to a value of $4.5 \mathrm{kOe}$ which might be related to the degradation of the interface quality for the thicker films as mentioned earlier. Following this assumption, a variation of the use magnetic anisotropy value with the film thickness might be expected resulting in a reduction of the coercive field as revealed by micromagnetic simulations. In addition, magnetostatic interaction between the neighboring caps will also cause a reduction in coercive field [54].

The evolution of the coercive field $H_{\mathrm{C}}$ and the width $\sigma$ of the SFD with the number of $[\mathrm{Co} / \mathrm{Pd}]$ bilayers in the multilayers grown on particle assemblies is summarized in Fig. (9c, d). The values of $H_{\mathrm{C}}$ and $\sigma$ are close to the ones reported by Thomson et al. [61] for lithographically patterned $[\mathrm{Co} / \mathrm{Pt}]$ multilayer films. The SFD is given as the full width at half maximum of the first derivative of the hysteresis loop fitted with a Gaussian distribution function. In addition to the intrinsic SFD arising from the inner defect structure of the $[\mathrm{Co} / \mathrm{Pd}]$ multilayers, the broadening of the SFD is given by the particle size distribution as well as by the influence of the dipolar fields coming from the neighboring caps $[61,62]$. In our case, the distribution of the particle sizes in the array plays initially the major role, however with increasing number of bilayers, the width $\sigma$ of the SFD increases linearly which is attributed to the effect of dipolar fields, which increases proportionally with the 

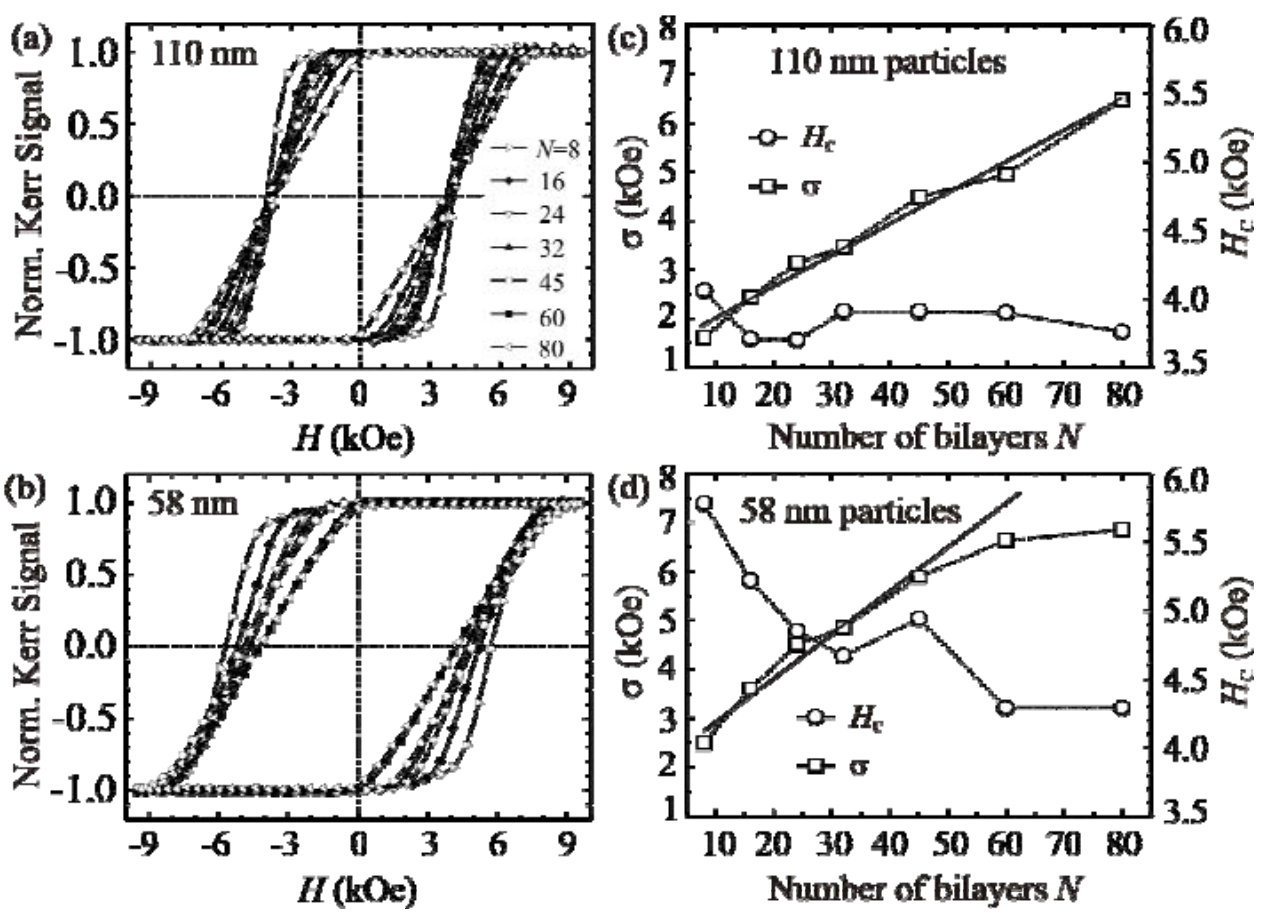

Fig. (9). Series of polar MOKE hysteresis loops of $[\mathrm{Co} / \mathrm{Pd}]_{\mathrm{N}}$ multilayer stacks with different bilayer number $N$ grown on (a) an assembly of $110 \mathrm{~nm}$ particles, and (b) an assembly of $58 \mathrm{~nm}$ particles. The evolution of switching field distribution, $\sigma$, and out-of-plane coercivity, $H_{\mathrm{C}}$, with bilayer number for $[\mathrm{Co} / \mathrm{Pd}]_{\mathrm{N}}$ samples grown on assemblies of (c) $110 \mathrm{~nm}$ and (d) $58 \mathrm{~nm}$ particles. Dotted lines are guides to the eye. Linear fits to the SFD data are also shown with solid lines. Reprinted with permission from Ulbrich et al. [43]. Copyright 2010, American Physical Society.

growing total magnetic moment of each nanocap. By comparing the evolution of the SFD with increasing bilayer number for the two particle arrays it becomes apparent that the SFD increases for the $58 \mathrm{~nm}$ array with a steeper slope (88 Oe per bilayer) than for the $110 \mathrm{~nm}$ array (64 Oe per bilayer), which is expected due to larger dipolar fields for smaller interparticle distances. Extrapolating the linear fit to the intersection with $y$-axis, we extracted the intrinsic SFD, $\sigma_{0}$, without magnetostatic contribution. As expected, owing to the larger particle size distribution, the value for $\sigma_{0}$ for the deposits on $58 \mathrm{~nm}$ particle arrays was found larger $(2.1 \mathrm{kOe})$ compared to the films grown on assemblies of $110 \mathrm{~nm}$ particles $(1.4 \mathrm{kOe})$. Interestingly the linear increase of the
SFD starts to level off when the total film thickness is in the range of the particle diameter $(N>50)$, thus probably indicating the onset of enhanced exchange coupling in the array.

Furthermore, MFM images taken on the samples show a well-defined black and white contrast on individual caps confirming that the caps are in a single domain state (Fig. 10). The domain size is independent of the number of bilayers, as the magnetic domains are localized at the cap structures even for $N=80$. This observation is quite surprising as the total film thickness is comparable to the particle diameter, where the issue of exchange coupling between caps may become important. However, despite the
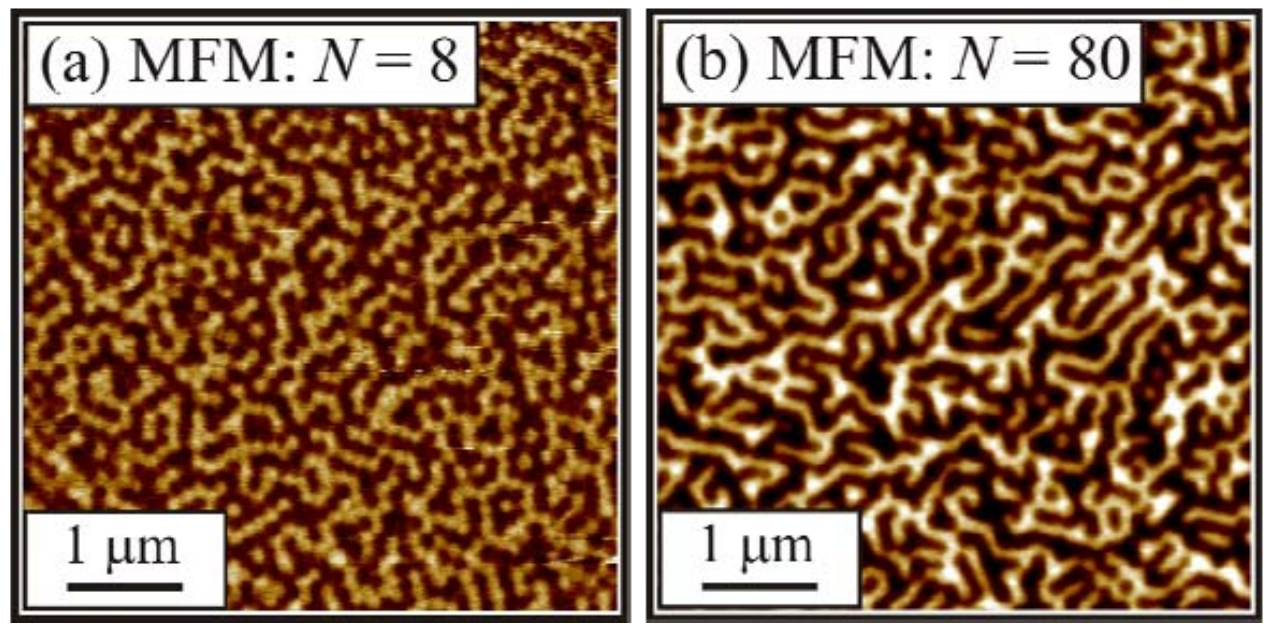

Fig. (10). MFM images taken on the samples with $N=8$ and 80 in the demagnetized state. Reprinted with permission from Ulbrich et al. [43]. Copyright 2010, American Physical Society. 
presence of exchange coupling throughout the array, individual switching events at cap positions can still occur followed by depinning of domain walls or further nucleation events depending on the intrinsic magnetic properties and the specific local magnetic environment. Similar results were reported for CoPt alloy films deposited onto particle arrays $[40,41]$.

\section{MAGNETIC ALLOYS ON ARRAYS OF SPHERICAL PARTICLES}

\subsection{Granular CoCrPt-SiO $\mathrm{Cll}_{2}$ Aloy Films on $\mathrm{SiO}_{2}$ Particle Arrays}

Granular $\mathrm{CoCrPt}-\mathrm{SiO}_{2}$ alloy films are used as storage material in conventional perpendicular magnetic recording systems [9]. Therefore the implementation of $\mathrm{CoCrPt}$ as a recording layer also for BPM was proposed [15, 63]. In this study, self-assembled arrays of $\mathrm{SiO}_{2}$ particles of various sizes were utilized to fabricate patterned media based on granular $\mathrm{CoCrPt}^{-\mathrm{SiO}_{2}}$ films [64]. Fig. (11) shows an MFM image of array of magnetic caps on particles with a size of $50 \mathrm{~nm}$ after $\mathrm{CoCrPt}-\mathrm{SiO}_{2}$ film deposition which was taken after demagnetizing the sample. It reveals bright and dark magnetic contrast located at the particle positions (see corresponding AFM image) confirming that the cap structures are in a magnetic single domain state. MFM images taken at remanence after saturating the samples show already reversed domains located on individual but randomly distributed caps.
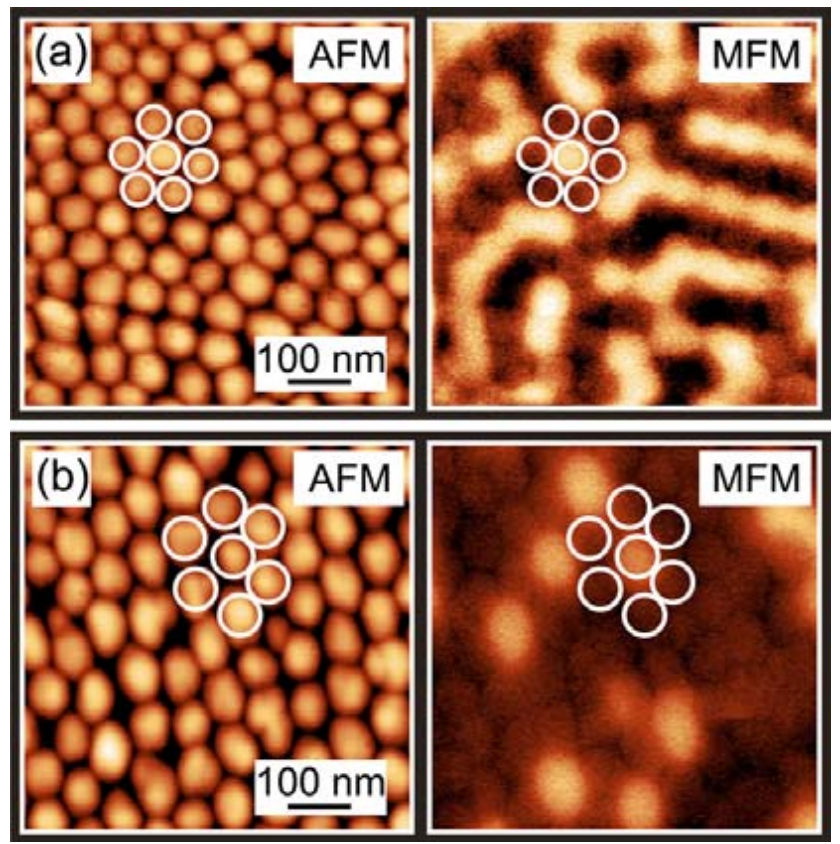

Fig. (11). AFM and corresponding MFM images of a CoCrPt$\mathrm{SiO}_{2} / \mathrm{Ru} / \mathrm{Ta}$ film grown on $\mathrm{SiO}_{2}$ particle array with a particle size of $50 \mathrm{~nm}$. Image (a) shows the magnetic domain configuration after demagnetizing the array of cap structures while image (b) reveals the magnetic contrast of individually reversed single domain caps at remanence after saturation. Reprinted with permission from Brombacher et al. [64]. Copyright 2010, American Institute of Physics.

Detailed Scanning Transmission Electron Microscopy (STEM) investigations have revealed a unique hedgehog-like morphology of small CoCrPt pillars which is induced by the curvature of the particle surface (Fig. 12a). Such a peculiar hedgehog-like cap structure can explain some of the distinct magnetic properties [64]. Starting with the observed reduction in the remanent magnetization after saturation, this can now be attributed to the distribution of the magnetic anisotropy axes of the columnar grains which are tilted following the curvature of the particle surface. Therefore, the magnetization at remanence in perpendicular orientation is expected to be lowered. Moreover, the presence of tilted magnetization orientations with respect to the applied field direction will also result in a reduced switching field which might be the reason that the demagnetization field at remanence is already sufficient to reverse the magnetization of individual caps.

Furthermore, Electron Energy Loss Spectroscopy (EELS) reveals the presence of a continuous $\mathrm{Co}$-rich layer underneath the CoCrPt pillars (Fig. 12b). This Co-rich layer is expected to give rise to enhanced intergranular exchange coupling of the granular film which results in the formation of single domain cap structures. It is interesting to note that the observed magnetic behavior in particular the angular dependence of the switching field is quite similar to the one measured on $[\mathrm{Co} / \mathrm{Pd}]_{\mathrm{N}}$ cap arrays even though the underlying microstructure is substantially different. However, both systems reveal a spatial distribution of the easy-axis pointing always perpendicular to the particle surface due to the interface anisotropy of the $[\mathrm{Co} / \mathrm{Pt}(\mathrm{Pd})]$ multilayers on the one hand and the columnar $\mathrm{CoCrPt}$ structure with tilted $c$ axis on the other hand. In addition, both systems are strongly exchange coupled (intercap coupling).

Recently, we demonstrated the possibility to tune the magnetic properties of $\mathrm{CoCrPt}-\mathrm{SiO}_{2}$ films grown on selfassembled arrays of $\mathrm{SiO}_{2}$ spherical particles using irradiation with magnetic $\mathrm{Co}^{+}$ions at fluences up to $4.5 \times 10^{14} \mathrm{~cm}^{-2}$ [63]. By using magnetic ions, we can directly influence the intergranular exchange by introducing Co atoms into the grain boundaries, which allows us to tailor the magnetic exchange coupling between $\mathrm{CoCrPt}$ grains in a controlled manner [65]. In particular, we found that irradiation at low fluences results in the increase of the exchange coupling leading to a substantial increase of the remanent magnetization. Furthermore, increase of the intergranular exchange coupling between $\mathrm{CoCrPt}$ grains initiated by $\mathrm{Co}^{+}$ irradiation enhances thermal stability of the magnetic media. However, irradiation at a larger fluence of $4.5 \times 10^{14} \mathrm{~cm}^{-2}$, the degradation of the magnetic layer becomes dominant and both, the remanent magnetization and the coercive field in the easy-axis direction decrease but does not show a pronounced dependence on the particle size. The degradation is assumed to be induced by the implantation of carbon from the top layer [66].

\subsection{FePt Films on $\mathrm{SiO}_{2}$ Particle Arrays}

To fulfill the requirements of thermal stability, CoCrPt as well as the $[\mathrm{Co} / \mathrm{Pt}(\mathrm{Pd})]_{\mathrm{N}}$ multilayer system are limited in their extensibility in storage density and thus Fe-Pt alloys in the $L 1_{0}$ chemically ordered phase are considered as candidate materials for a recording layer in BPM allowing densities beyond 1 Tbit/inch ${ }^{2}$ [12]. However, several important aspects have to be considered: (i) Chemical $L 1_{0}$ ordering is 
required to induce strong magnetic anisotropy and (ii) (001) texturing of the FePt film is needed to stabilize PMA. While the first issue can be solved by thermal treatment of the alloys, the second property is more difficult to achieve. Please note that conventional annealing of FePt films grown on amorphous substrates results in a chemically ordered alloy, but with isotropic orientation of the easy axes of magnetization [67]. Therefore, single crystalline substrates with appropriate orientation like $\mathrm{MgO}(001)$ or $\mathrm{NaCl}(001)$ can be used to stabilize a (001) texture in FePt alloys via epitaxial growth $[12,68]$.

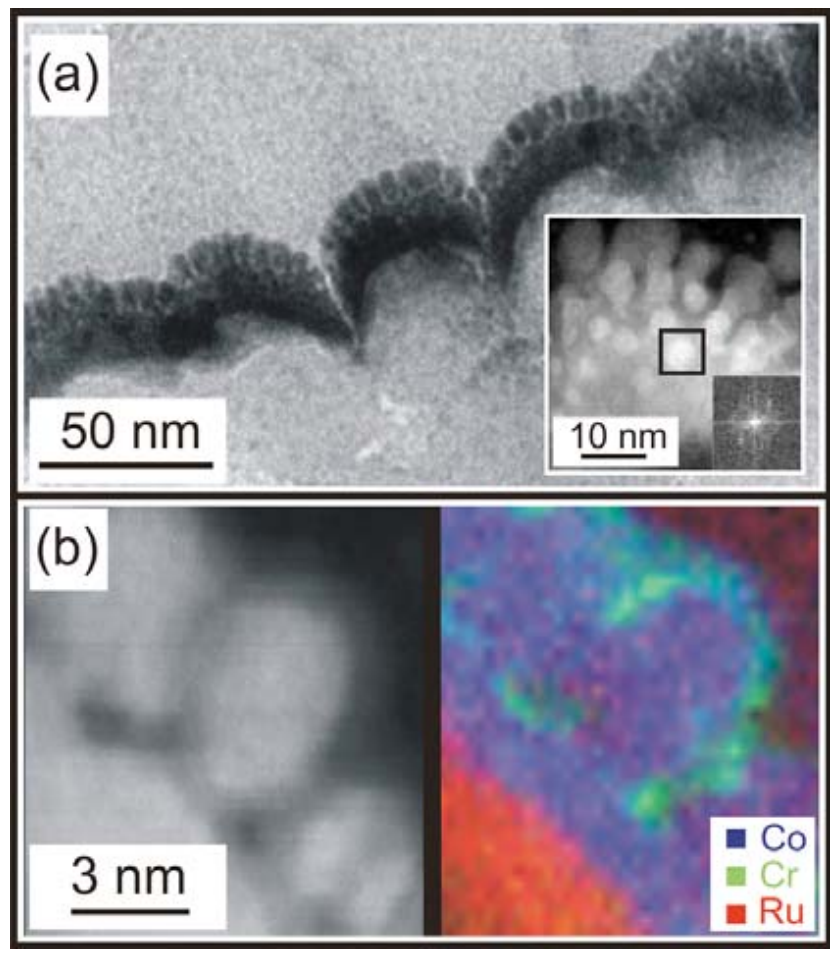

Fig. (12). (a) Sample overview of a $\mathrm{CoCrPt}-\mathrm{SiO}_{2} / \mathrm{Ru} / \mathrm{Ta}$ film grown on a $\mathrm{SiO}_{2}$ particle array with particle size of $50 \mathrm{~nm}$ using bright field TEM imaging. The inset reveals details of the hedgehog shape with the corresponding Fourier transformation of one marked nanopillar showing threefold symmetry. (b) Bright field TEM image and corresponding elemental mapping. Reprinted with permission from Brombacher et al. [64]. Copyright 2010, American Institute of Physics.

In this regard, we used $[\operatorname{Pt}(3 \mathrm{~nm}) / \operatorname{Cr}(50 \mathrm{~nm})]$ seed layers which were deposited at $350^{\circ} \mathrm{C}$ onto assemblies of nonmagnetic $\mathrm{SiO}_{2}$ particles to initiate the (001) texture in 5nm-thick FePt alloy films [39]. Fig. (13) shows polar MOKE hysteresis loops measured on the samples grown on arrays with particle sizes of $160 \mathrm{~nm}$ and $330 \mathrm{~nm}$. Larger coercive fields of about $370 \mathrm{mT}$ and a broader SFD compared to the continuous film (open symbols) was obtained. While a remanence magnetization $\left(M_{\mathrm{R}} / M_{\mathrm{S}}\right.$ ratio) of almost 1 is obtained for the film on $330 \mathrm{~nm}$ particle arrays, a reduced value of 0.7 is observed for the magnetic caps on $160 \mathrm{~nm}$ particles. The latter clearly indicates that nucleation and reversal of the domains starts already before remanence when first saturated.

The high value of coercivity and the substantially reduced remanent magnetization for the FePt alloy deposited on array of $160 \mathrm{~nm}$ particles is an indication of a still large value of the chemical order parameter, but with a different texture compared to the sample on $330 \mathrm{~nm}$ particles. In order to clarify this point, a detailed structural characterization of the film stack on $160 \mathrm{~nm}$ particle was performed by TEM (Fig. 14). A drastic decrease in the grain size down to $7 \mathrm{~nm}$ compared to the film grown on a planar $\mathrm{SiO}_{2}$ substrate is visible. The grains grow perpendicular to the sphere surface even at high angles and grains of the neighboring particles are effectively interconnected enhancing the magnetic exchange coupling between the magnetic caps. The grain structure is also columnar like on a planar film, but the interfaces between the grains are much wider $(2-3) \mathrm{nm}$ (Fig. 14). The grains in the FePt layer have rounded caps which fall off rapidly to the sides (cf. dotted line in Fig. (14b)). The bcc $\mathrm{Cr}$ layer has a (110) orientation resulting in a (111) texture in the FePt layer. A larger influence of strain for smaller particles is considered to be responsible for the different structural properties of the $\mathrm{Cr}$ layer when grown onto spheres with a size of 160 and $330 \mathrm{~nm}$. Therefore, further optimization of the structural properties of the $\mathrm{Cr} / \mathrm{Pt}$ bilayer is needed to achieve the required (001) texture in FePt alloys.

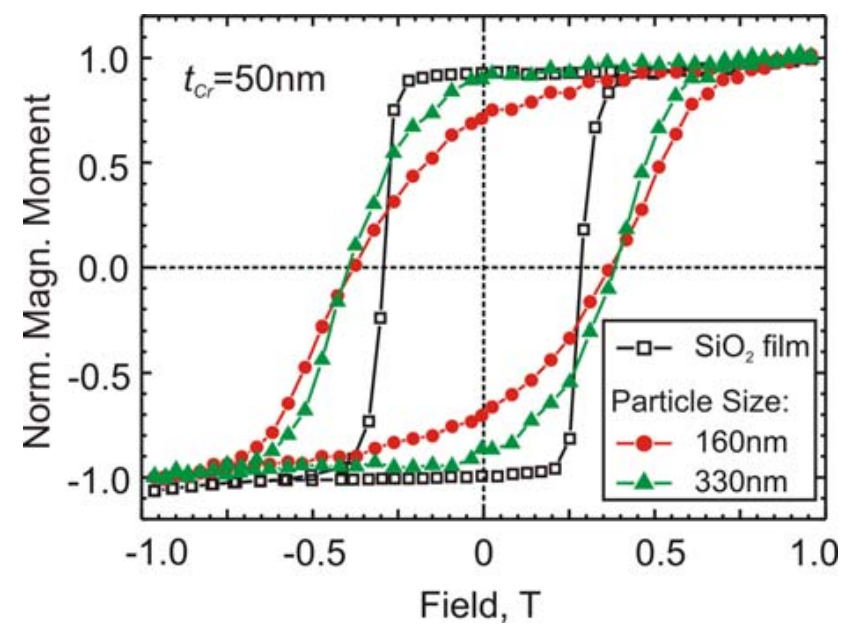

Fig. (13). $\mathrm{FePt}\left(5 \mathrm{~nm} @ 450^{\circ} \mathrm{C}\right) / \operatorname{Pt}\left(3 \mathrm{~nm} @ 350^{\circ} \mathrm{C}\right) / \mathrm{Cr}(50 \mathrm{~nm} @$ $350^{\circ} \mathrm{C}$ ) on arrays of $\mathrm{SiO}_{2}$ particles. Polar MOKE hysteresis loops of the samples grown on arrays of $160 \mathrm{~nm}$ (red circles) and $330 \mathrm{~nm}$ (green triangles) $\mathrm{SiO}_{2}$ spheres. The hysteresis loop of the sample grown on a planar substrate is presented for comparison (open symbols). Reprinted with permission from Makarov et al. [39]. Copyright 2008, American Institute of Physics.

\section{MAGNETIC CAP STRUCTURES FOR DATA STORAGE}

In addition to the issues of fabricating ordered arrays of magnetic nanostructures over large areas, methods need to be found for successful writing of information in to the bit patterned media. Writing imposes a careful lateral adjustment of the recording head position on the track and requires the synchronization of write pulse to the media pattern [69-71]. For this probe recording scheme, a scanning tunneling microscopy or an atomic force microscopy cantilever tip coated with a magnetic material used to $\mathrm{read} / \mathrm{write}$ information to each magnetic nanostructure is considered to be promising [72]. Probe recording has been 


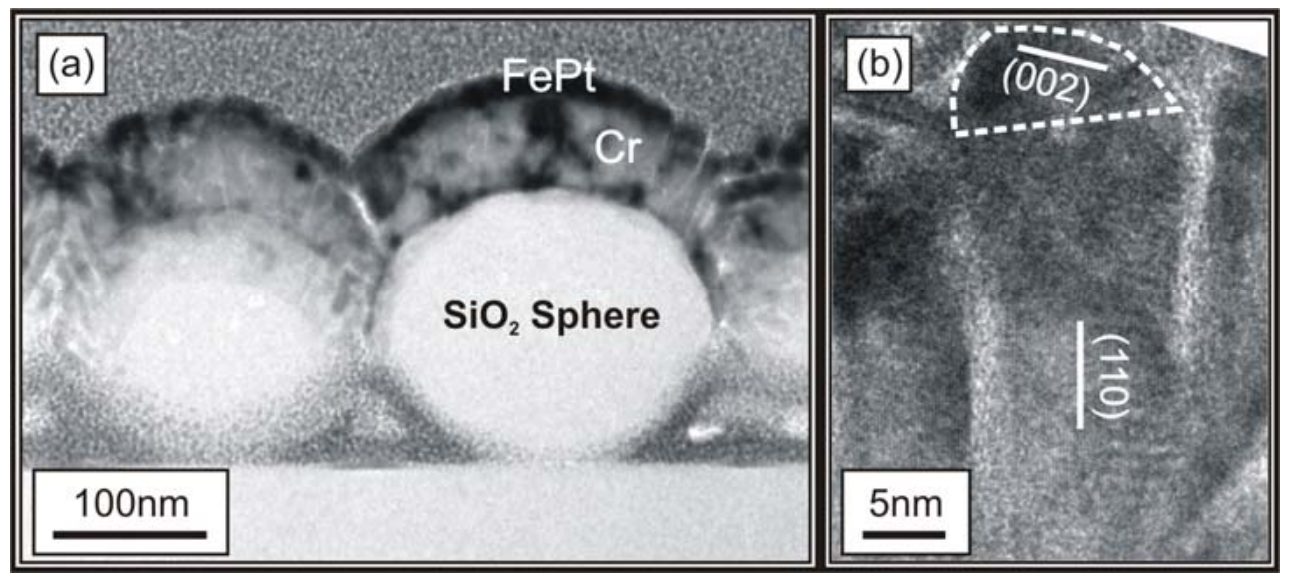

Fig. (14). $\mathrm{FePt}\left(5 \mathrm{~nm} @ 450^{\circ} \mathrm{C}\right) / \mathrm{Pt}\left(3 \mathrm{~nm} @ 350^{\circ} \mathrm{C}\right) / \mathrm{Cr}\left(50 \mathrm{~nm} @ 350^{\circ} \mathrm{C}\right)$ on $160 \mathrm{~nm} \mathrm{SiO} \mathrm{S}_{2}$ particles. (a) Cross-sectional bright field TEM image. (b) High resolution TEM micrograph of the grain structure of FePt layer and the underlayer on the particle. Reprinted with permission from Makarov et al. [39]. Copyright 2008, American Institute of Physics.

achieved in nonmagnetic materials by oxidizing the surface of the storage material, by charge storage in semiconductors or by forming indentations in polymers [73, 74]. However, these techniques suffer from the relatively slow reading and writing speed. Therefore, large scale parallelization of the tips is required to boost performance as demonstrated for example in the Millipede system [72, 73]. Probe recording on magnetic materials requires for instance the use of magnetic force microscopy (MFM) tips.

In order to perform probe recording on arrays of magnetic cap structures, MFM tips have to fulfill two critical requirements: The stray field of the MFM tip has to be sufficiently large to reverse the magnetization direction of the individual magnetic cap and the spatial distribution of the stray field has to be rather narrow (i.e. the stray field gradient has to be sufficiently steep) so that the magnetic state of the neighboring magnetic nanostructures is not affected. The stray field of less than $10 \mathrm{mT}$ of commercially available MFM tips is generally too weak for probe recording applications. Therefore, in order to increase the tip's stray field, a series of MFM tips was specially manufactured [42]. The largest stray field of $67.5 \mathrm{mT}$ was achieved by using a $400 \mathrm{~nm}$ long Co rod with a diameter of $100 \mathrm{~nm}$ grown by electrochemical deposition inside an aluminum membrane and attached near the apex of the cantilever tip. To focus its stray field, the rod's apex was further shaped into a cone by focused ion beam (inset in Fig. (15d)) resulting in an apex diameter of less than $25 \mathrm{~nm}$.

In this study, template-directed self-assembly process was employed to arrange spherical particles into a polymer resist template with regular hole arrays, produced using extreme ultraviolet interference lithography [42] as already presented in Fig. (4). An array of magnetic caps was created by depositing $[\operatorname{Pt}(0.8 \mathrm{~nm}) / \mathrm{Co}(0.3 \mathrm{~nm})]_{8} / \mathrm{Pt}(5 \mathrm{~nm})$ multilayers onto these patterns forming single domain cap structures with out-of-plane magnetization.

The proof-of-concept probe recording measurements were performed using a variable temperature UHV-MFM at a base temperature of $8 \mathrm{~K}$. The magnetic state of the sample in an applied bias magnetic field is presented in Fig. (15a) revealing that the sample is almost magnetically saturated with only a few magnetic caps left with a reverse magnetization (in white) which we use for the probe recording trials. In order to initiate a magnetic writing event, the MFM tip was positioned over the nanocap of choice. Then a force-distance curve was acquired by approaching the cantilever slowly in vertical direction (Fig. 15d), red solid curve). The spectra shows a well-pronounced peak at a tipsample separation distance of about $1.2 \mathrm{~nm}$, indicating a magnetic switching event (island in red circular frame in Fig. (15a)), which is confirmed by the next MFM scan performed at larger tip-sample separation (Fig. 15b). Moreover, in an additional measurement taken after the switching event at the same location (Fig. 15d), black dashed curve) no peak is present. Indeed, the presence of the peak in a force-distance curve is useful for tracking successful switching events in a magnetic probe recording concept. In addition, two further switching events are indicated by the blue square frames in Fig. (15b, c), clearly demonstrating that using the field assisted probe recording approach, isolated single nanocaps can be switched in a controlled fashion.

\section{CONCLUSION}

Magnetic and structural properties of magnetic thin films grown onto assemblies of nonmagnetic spherical particles are reviewed. As the size of the particles as well as the thickness of the deposited magnetic film can be tuned at will, the so fabricated magnetic cap structures represent a convenient system to study the fundamental magnetic interactions (exchange and magnetostatic) at the nanoscale. Due to the curvature of the particle's surface, magnetic and structural properties of the magnetic thin films differ substantially from their planar counterparts with respect to the equilibrium magnetic domain pattern as well as magnetization reversal behavior.

Furthermore, these magnetic cap structures were attempted to create a bit patterned magnetic media for magnetic data storage applications. In this respect, several challenges still need to be addressed in order to realize a BPM recording system. On the one hand, techniques for fabricating ordered arrays of magnetic nanostructures over large areas are needed without degradation of the 


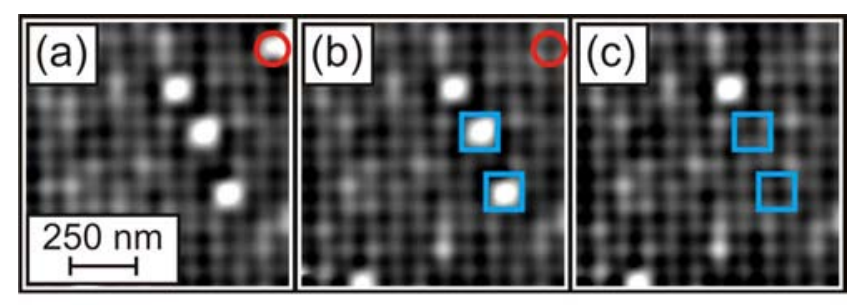

(d)

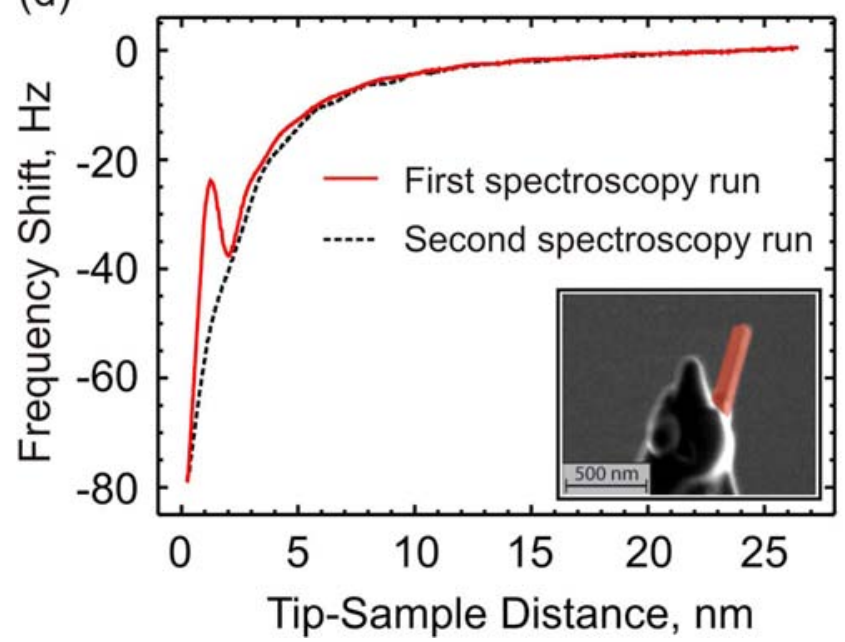

Fig. (15). Probe recording on an array of nanocaps on $60 \mathrm{~nm}$ nanoparticles with a period of $100 \mathrm{~nm}$. (a-c) The series of MFM images reveals that the magnetization of the dots (marked with a frame) switch by the tip field from an antiparallel (bright) to a parallel configuration (dark). The graph in (d) shows force-distance curves displaying a kink when the magnetization of the nanocap changes its direction. A custom made rod-like tip used for probe recording experiments is shown as an inset in (d). Reprinted with permission from Kappenberger et al. [42]. Copyright 2009, American Institute of Physics.

perpendicular magnetic anisotropy towards smaller bit size; on the other hand, methods need to be found for successful writing of information in the BPM. For the latter, there are two potentially interesting approaches to reduce the switching field: (i) heat assisted magnetic recording (HAMR) and (ii) exchange coupled composite (ECC) media. HAMR relies on the local heating of the magnetic nanostructure close to its Curie temperature just before or during the writing event $[75,76]$. A different route is envisioned in the ECC media concept [77-79]. Here, a magnetic nanostructure or bit consists of two magnetic components: a soft and a hard magnetic part. In this composite, the thermal stability is given by the hard component, while its switching field and switching field distribution can be substantially reduced due to the soft part [80]. Both concepts are currently intensively investigated offering new advances in achieving higher densities following 'Moore's Law' in future storage devices.

\section{ACKNOWLEDGEMENT}

Declared none.

\section{CONFLICT OF INTEREST}

The authors confirm that this article content has no conflicts of interest.

\section{REFERENCES}

[1] Ono A, Kato J, Kawata S. Subwavelength optical imaging through a metallic nanorod array. Phys Rev Lett 2005; 95(26): 267407.

[2] Huynh WU, Dittmer JJ, Alivizatos AP. Hybrid nanorod-polymer solar cells. Science 2002; 295(5564): 2425-7.

[3] Huang YF, Chang HT, Tan W. Cancer cell targeting using multiple aptamers conjugated on nanorods. Anal Chem 2008; 80(3): 567-72.

[4] Fu J, Park BB, Siragusa G, et al. An Au/Si hetero-nanorod-based biosensor for Salmonella detection. Nanotechnology 2008; 19(15): 155502.

[5] Xue SH, Wang ZD. Metal nanorod arrays and their magnetic properties. Mater Sci Eng B 2006; 135(1): 74-7.

[6] Ross CA. Patterned magnetic recording media. Ann Rev Mater Sci 2001; 31: 203-35.

[7] Sun S, Murray CB, Weller D, et al. Monodisperse FePt nanoparticles and ferromagnetic FePt nanocrystal superlattices. Science 2000; 287(5460): 1989-92.

[8] Terris BD, Thomson T. Nanofabricated and self-assembled magnetic structures as data storage media. J Phys D: Appl Phys 2005; 38(12): R199-R222.

[9] Piramanayagam SN. Perpendicular recording media for hard disk drives. J Appl Phys 2007; 102(1): 011301.

[10] Lancelle-Beltran E, Prene P, Boscher C, et al. Nanostructured hybrid solar cells based on self-assembled mesoporous titania thin films. Chem Mater 2006; 18(26): 6152-6.

[11] Baeumner AJ. Nanosensors identify pathogens in food. Food Technol 2004; 58(8): 51-5.

[12] Piramayagam SN, Srinivasan K. Recording media research for future hard disk drives. J Magn Magn Mater 2009; 321(6): 485-94.

[13] Weller D, Moser A. Thermal effect limits in ultrahigh density magnetic recording. IEEE Trans Magn 1999; 35(6): 4423-39.

[14] Greaves SJ, Muraoka H, Kanai Y. Discrete track media for 600 Gbits/in ${ }^{2}$ recording. J Appl Phys 2006; 99(8): 08F903.

[15] Terris BD. Fabrication challenges for patterned recording media. J Magn Magn Mater 2009; 321(6): 512-7.

[16] Wood R. Future hard disk drive systems. J Magn Magn Mater 2009; 321(6): 555-61.

[17] Lyubina J, Rellinghaus B, Gutfleisch O, Albrecht M. Structure and Magnetic Properties of $\mathrm{L1}_{0}$-Ordered $\mathrm{FePt}$ Alloys and Nanoparticles, In: Buschow KHJ, Ed. Handbook of Magnetic Materials. UK: Elsevier 2011; vol. 19: pp. 291-395.

[18] Farrow RFC, Weller D, Marks RF, et al. Control of the axis of chemical ordering and magnetic anisotropy in epitaxial FePt films. J Appl Phys 1996; 79(8): 5967.

[19] Weller D, Brändle H, Gorman G, et al. Magnetic and magnetooptical properties of cobalt/platinum alloys with perpendicular magnetic anisotropy. Appl Phys Lett 1992; 61(22): 2726.

[20] $\mathrm{Hu} \mathrm{G}$, Thomson T, Albrecht M, et al. Magnetic and recording properties of $\mathrm{Co} / \mathrm{Pd}$ islands on prepatterned substrates. J Appl Phys 2004; 95(11): 7013 .

[21] Lodder JC. Methods for preparing patterned media for high-density recording. J Magn Magn Mater 2004; 272-276(3): 1692-7.

[22] Hyndman R, Warin P, Gierak J, et al. Modification of $\mathrm{Co} / \mathrm{Pt}$ multilayers by gallium irradiation-Part 1: The effect on structural and magnetic properties. J Appl Phys 2001; 90(8): 3843.

[23] Luo F, Heyderman LJ, Solak HH, et al. Nanoscale perpendicular magnetic island arrays fabricated by extreme ultraviolet interference lithography. Appl Phys Lett 2008; 92(10): 102505.

[24] McClelland GM, Hart MW, Rettner CT, et al. Nanoscale patterning of magnetic islands by imprint lithography using a flexible mold. Appl Phys Lett 2002; 81(8): 1483.

[25] Grobis M, Dobisz E, Hellwig O, et al. Measurements of the write error rate in bit patterned magnetic recording at $100-320 \mathrm{~Gb} / \mathrm{in}^{2}$. Appl Phys Lett 2010; 96(5): 052509.

[26] Wang T, Wang Y, Fu Y, et al. Magnetic behavior in an ordered Co nanorod array. Nanotechnology 2008; 19(45): 455703.

[27] Walcarius A, Sibottier E, Etienne M, et al. Electrochemically assisted self-assembly of mesoporous silica thin films. Nat Mater 2007; 6: 602-8.

[28] Fisher A, Kuemmel M, Järn M, et al. Surface Nanopatterning by Organic/Inorganic Self-Assembly and Selective Local Functionalization. Small 2006; 2(4): 569-74.

[29] Grosso D, Boissière C, Smarsly B, et al. Periodically ordered nanoscale islands and mesoporous films composed of nanocrystalline multimetallic oxides. Nat Mater 2004; 3: 787-92. 
[30] Makarov D, Krone P, Lantiat D, et al. Magnetization Reversal in Arrays of Magnetic Nanoperforations. IEEE Trans Magn 2009; 45(10): 3515-8.

[31] Schulze C, Faustini M, Lee J, et al. Magnetic films on nanoperforated templates: a route towards percolated perpendicular media. Nanotechnology 2010; 21(49): 495701.

[32] Grobis M, Schulze C, Faustini M, et al. Recording study of percolated perpendicular media. Appl Phys Lett 2011; 98(19): 192504.

[33] Bita I, Yang JKW, Jung YS, et al. Graphoepitaxy of self-assembled block copolymers on two-dimensional periodic patterned templates. Science 2008; 321: 939-43.

[34] Hellwig O, Bosworth JK, Dobisz E, et al. Bit patterned media based on block copolymer directed assembly with narrow magnetic switching field distribution. Appl Phys Lett 2010; 96(5): 052511.

[35] Facsko S, Dekorsy T, Koerdt C, et al. Formation of ordered nanoscale semiconductor dots by ion sputtering. Science 1999; 285: 1551-3.

[36] Zhu M, Li Y, Meng T, et al. Controlled strain on a doubletemplated textured polymer film: a new approach to patterned surfaces with bravais lattices and chains. Langmuir 2006; 22(17): 7248-53.

[37] Albrecht $\mathrm{M}, \mathrm{Hu} \mathrm{G}, \mathrm{Guhr}$ IL, et al. Magnetic multilayers on nanospheres. Nat Mater 2005; 4: 203-6.

[38] Ulbrich TC, Makarov D, Hu G, et al. Magnetization reversal in a novel gradient nanomaterial. Phys Rev Lett 2006; 96(7): 077202.

[39] Makarov D, Brombacher C, Liscio F, et al. FePt films on selfassembled $\mathrm{SiO}_{2}$ particle arrays. J Appl Phys 2008; 103(5): 053903.

[40] Makarov D, Bermúdez-Ureña E, Schmidt OG, et al. Nanopatterned CoPt alloys with perpendicular magnetic anisotropy. Appl Phys Lett 2008; 93(15): 153112.

[41] Makarov D, Klimenta F, Fischer S, et al. Nonepitaxially grown nanopatterned Co-Pt alloys with out-of-plane magnetic anisotropy. J Appl Phys 2009; 106(11): 114322.

[42] Kappenberger P, Luo F, Heyderman LJ, et al. Template-directed self-assembled magnetic nanostructures for probe recording. Appl Phys Lett 2009; 95(2): 023116.

[43] Ulbrich TC, Bran C, Makarov D, et al. Effect of magnetic coupling on the magnetization reversal in arrays of magnetic nanocaps. Phys Rev B 2010; 81(5): 054421.

[44] Günther CM, Hellwig O, Menzel A, et al. Microscopic reversal behavior of magnetically capped nanospheres. Phys Rev B 2010; 81(6): 064411.

[45] Micheletto R, Fukuda H, Ohtsu M. A simple method for the production of a two-dimensional, ordered array of small latex particles. Langmuir 1995; 11(9): 3333-6.

[46] Carcia PF, Meinhaldt AD, Suna A. Perpendicular magnetic anisotropy in $\mathrm{Pd} / \mathrm{Co}$ thin film layered structures. Appl Phys Lett 1985; 47(2): 178.

[47] Hashimoto S, Ochiai Y, Aso K. Perpendicular magnetic anisotropy and magnetostriction of sputtered $\mathrm{Co} / \mathrm{Pd}$ and $\mathrm{Co} / \mathrm{Pt}$ multilayered films. J Appl Phys 1989; 66(10): 4909.

[48] Lin C-J, Gorman GL, Lee CH. Magnetic and structural properties of Co/Pt multilayers. J Magn Magn Mat 1991; 93: 194-206.

[49] Kim J-H, Shin S-C. Interface roughness effects on the surface anisotropy in Co/Pt multilayer films. J Appl Phys 1996; 80(5): 3121.

[50] Guo VW, Lu B, Wu X, et al. A survey of anisotropy measurement techniques and study of thickness effect on interfacial and volume anisotropies in Co/Pt multilayer media. J Appl Phys 2006; 99(8): 08E918.

[51] Stoner EC, Wohlfarth EP. A mechanism of magnetic hysteresis in heterogeneous alloys. Trans R Soc A 1948; 240(826): 599-642.

[52] Ulbrich TC, Assmann D, Albrecht M. Magnetic properties of $\mathrm{Co} / \mathrm{Pt}$ multilayers on self-assembled particle arrays. J Appl Phys 2008; 104(8): 084311.

[53] Weller D, Folks L, Best M, et al. Growth, structural, and magnetic properties of high coercivity Co/Pt multilayers. J Appl Phys 2001; 89(11): 7525 .
[54] Krone P, Makarov D, Schrefl T, et al. Effect of the anisotropy distribution on the coercive field and switching field distribution of bit patterned media. J Appl Phys 2009; 106(10): 103913.

[55] Soares MM, de Biasi E, et al. Magnetic vortices in tridimensional nanomagnetic caps observed using transmission electron microscopy and magnetic force microscopy. Phys Rev B 2008; 77(22): 224405.

[56] den Broeder FJA, Hoving W, Bloemen PJH. Magnetic anisotropy of multilayers. J Magn Magn Mater 1991; 93: 562-70.

[57] Engel BN, England CD, Van Leeuwen RA, et al. Interface magnetic anisotropy in epitaxial superlattices. Phys Rev Lett 1991; 67(14): 1910-3.

[58] Bagguley DMS, Crossley WA, Liesegang J. Ferromagnetic resonance in a series of alloys: II. Binary alloys of cobalt with platinum and palladium, and one iron-palladium alloy. Proc Phys Soc 1967; 90(4): 1047-58.

[59] Childress JR, Duvail JL, Jasmin S, et al. Perpendicular magnetic anisotropy in $\mathrm{Co}_{\mathbf{x}} \mathrm{Pd}_{1-\mathrm{x}}$ alloy films grown by molecular beam epitaxy. J Appl Phys 1994; 75(10): 6412.

[60] Kimling née Moser J, Kunej V, Pernau H, et al. Magnetoresistive effects in $\mathrm{Co} / \mathrm{Pd}$ multilayers on self-assembled nanoparticles. J Appl Phys 2010; 107(9): 09C506.

[61] Thomson T, Hu G, Terris BD. Intrinsic distribution of magnetic anisotropy in thin films probed by patterned nanostructures. Phys Rev Lett 2006; 96(25): 257204.

[62] Hellwig O, Berger A, Thomson T, et al. Separating dipolar broadening from the intrinsic switching field distribution in perpendicular patterned media. Appl Phys Lett 2007; 90(16): 162516.

[63] Krone P, Brombacher C, Makarov D, et al. Nanocap arrays of granular $\mathrm{CoCrPt}: \mathrm{SiO}_{2}$ films on silica particles: tailoring of the magnetic properties by $\mathrm{Co}^{+}$irradiation. Nanotechnology 2010; 21(38): 385703.

[64] Brombacher C, Falke M, Springer F, et al. Magnetic hedgehog-like nanostructures. Appl Phys Lett 2010; 97(10): 102508.

[65] Tibus S, Strache T, Springer F, et al. Magnetic properties of granular $\mathrm{CoCrPt}: \mathrm{SiO}_{2}$ films as tailored by $\mathrm{Co}+$ irradiation. J Appl Phys 2010; 107(9): 093915.

[66] Rettner CT, Anders S, Thomson T, et al. Magnetic characterization and recording properties of patterned $\mathrm{CO}_{70} \mathrm{Cr}_{18} \mathrm{Pt}_{12}$ perpendicular media. IEEE Trans Magn 2002; 38(4): 1725-30.

[67] Takahashi YK, Seki TO, Hono K, et al. Microstructure and magnetic properties of $\mathrm{FePt}$ and $\mathrm{Fe} / \mathrm{FePt}$ polycrystalline films with high coercivity. J Appl Phys 2004; 96(1): 475.

[68] Liscio F, Makarov D, Maret M, et al. Growth, structure and magnetic properties of $\mathrm{FePt}$ nanostructures on $\mathrm{NaCl}(001)$ and $\mathrm{MgO}(001)$. Nanotechnology 2010; 21(6): 065602.

[69] Lohau J, Moser A, Rettner CT, et al. Writing and reading perpendicular magnetic recording media patterned by a focused ion beam. Appl Phys Lett 2001; 78(7): 990.

[70] Barbic M, Schultz S, Wong J, et al. Recording processes in perpendicular patterned media using longitudinal magnetic recording heads. IEEE Trans Magn 2001; 37(4): 1657-60.

[71] Albrecht M, Rettner CT, Moser A, et al. Magnetic reversal of sub$100 \mathrm{~nm}$ nanostructures studied by a FIB-trimmed recording head. Microsyst Technol 2007; 13: 129-32.

[72] Mamin HJ, Terris BD, Fan L-S, et al. High-density data storage using proximal probe techniques. IBM J Res Develop 1995; 39: 681-700.

[73] Vettiger P, Cross G, Despont M, et al. The "millipede" - nanotechnology entering data storage. IEEE Trans. Nanotechnology 2002; 1(1): 39-55.

[74] Gidon S, Lemonnier O, Rolland B, et al. Electrical probe storage using Joule heating in phase change media. Appl Phys Lett 2004; 85(26): 6392.

[75] Challener WA, Peng C, Itagi AV, et al. Heat-assisted magnetic recording by a near-field transducer with efficient optical energy transfer. Nat Photonics 2009; 3: 220-4. 
[76] Stipe BC, Strand TC, Poon CC, et al. Magnetic recording at 1.5 $\mathrm{Pb} / \mathrm{m}^{2}$ using an integrated plasmonic antenna. Nat Photonics 2010; 4: 484-8.

[77] Suess D, Fidler J, Porath K, et al. Micromagnetic study of pinning behavior in percolated media. J Appl Phys 2006; 99(8): 08G905.
[78] Suess D, Lee J, Fidler J, et al. Exchange-coupled perpendicular media. J Magn Magn Mater 2009; 321(6): 545-54.

[79] Victora RH, Shen X. Exchange coupled composite media. Proc IEEE 2008; 96(11): 1799-809.

[80] Krone P, Makarov D, Schrefl T, et al. Exchange coupled composite bit patterned media. Appl Phys Lett 2010; 97(8): 082501.

Received: January 27, 2011

Revised: October 6, 2011

Accepted: February 1, 2012

(C) Albrecht and Makarov; Licensee Bentham Open.

This is an open access article licensed under the terms of the Creative Commons Attribution Non-Commercial License (http: //creativecommons.org/licenses/by$\mathrm{nc} / 3.0 /$ ) which permits unrestricted, non-commercial use, distribution and reproduction in any medium, provided the work is properly cited. 